\title{
Estrias angióides: análise fundoscópica de 317 casos
}

\author{
Angioid streaks: fundoscopic analysis of 317 cases
}

\author{
Suel Abujamra ${ }^{1}$ \\ Alan Diego Negretto ${ }^{2}$ \\ Janaína Jamile Ferreira Saraceno ${ }^{3}$ \\ Tâmara Lopes Oliveira ${ }^{4}$ \\ André Marcelo Vieira Gomes ${ }^{2}$
}

\begin{tabular}{l} 
RESUMO \\
\hline Objetivo: Avaliar a presença de neovascularização de coróide (NVC) e \\
suas seqüelas em pacientes portadores de estrias angióides (EA). Méto- \\
dos: Foram analisados retrospectivamente os prontuários de pacientes \\
atendidos no Instituto Suel Abujamra, São Paulo (SP), Brasil, de 1976 a \\
2006. Considerou-se a idade, cor da pele, queixas na primeira consulta, \\
acuidade visual e aspecto fundoscópico com especial atenção a lesões \\
disciformes por NVC sub-retinianas ativas ou cicatrizadas. Resultados: \\
Dos 317 pacientes estudados, 163(51,5\%) eram homens. A média de idade \\
era de 57 anos com desvio-padrão de 12,4 anos, mínimo de 11 e máximo de \\
91 anos. Quanto a cor da pele, 209 (66,2\%) eram brancos, 91 (29,0\%) \\
amarelos,11 (3,7\%) negros, e 3 (1,1\%) pardos. As EA eram bilaterais em \\
todos os pacientes, e de 631 olhos pesquisados, 348 (55,1\%) tinham EA de \\
coloraçãomarrom, 153 (24,2\%) cinza e 124(19,6\%) marrom-avermelhada. \\
No momento do diagnóstico, as acuidades visuais medidas na tabela de \\
Snellen foram piores que 20/200 em 40,4\% dos olhos. Comprovou-se a \\
ausência de NVC em 103 (32,5\%) pacientes. As lesões NVC unilaterais \\
estavam presentes em 112 (35,3\%) e bilaterais em 99 (31,2\%). Quanto à \\
localização, 242 (74,3\%) eram maculares, 45 (13,8\%) extra-maculares e \\
38 (11,7\%) mistas. Conclusão: As EA são importante causa de cegueira \\
legal e podem passar despercebidas no exame oftalmológico de rotina \\
quando não apresentamlesões maculares disciformes ativas ou cicatrizadas. \\
A importância deste estudo é o expressivo número de casos e alertar os \\
oftalmologistas quanto à necessidade de um exame fundoscópico minu- \\
cioso, a fim de monitorá-las, diagnosticar e tratar precocemente a NVC. \\
\hline
\end{tabular}

Descritores: Estrias angióides; Doenças retinianas; Retina/patologia

\section{INTRODUÇÃO}

Estrias angióides (EA) são deiscências da camada elástica da membrana de Bruch secundárias a uma fragilidade anormal de seu tecido elástico ${ }^{(1)}$. Faz parte de um processo degenerativo de elastorexis universal.

Oftalmoscopicamente, apresenta-se como bandas marrom-acizentadas, múltiplas ou únicas, bilaterais, assimétricas, dispostas de forma radial a partir do nervo óptico.

Achados associados incluem alterações pigmentares com fundus moteado (peau d'orange), drusas de nervo óptico, drusas do epitélio pigmentar da retina, cicatrizes retinianas periféricas e calcificações ${ }^{(2)}$. Podem estar associadas à doenças sistêmicas como o pseudoxantoma elástico, doença de Paget, doença de Ehlers-Danlos e hemoglobinopatias.

$\mathrm{O}$ fato das estrias poderem se aproximar à fóvea e seu comprimento poder atingir todo o pólo posterior faz com que a mácula seja mais suscetível ao desenvolvimento de neovascularização de coróide (NVC). Esta 
complicação ocorre em 72 a $86 \%$ dos pacientes ${ }^{(3)}$, tornando-se bilaterais em metade deles dentro de 18 meses $^{(4)}$.

Apesar de serem descritas inicialmente por Doyne ${ }^{(5)} \mathrm{em}$ 1889, as EA têm ainda, nos dias atuais, suas modalidades terapêuticas limitadas, a ponto de muitos pacientes estarem legalmente cegos por volta dos $50 \operatorname{anos}^{(6)}$.

Os objetivos desta análise foram descrever os aspectos epidemiológicos ao longo de trinta anos no estudo das EA em nossa instituição.

\section{MÉTODOS}

Num estudo institucional, retrospectivo, observacional e descritivo, foram analisados os prontuários de 317 pacientes atendidos no Instituto Suel Abujamra, São Paulo (SP), Brasil, de 1976 a 2006 com diagnóstico de EA.

O exame oftalmológico no primeiro atendimento constava de acuidade visual corrigida, biomicroscopia de fundo, pressão intra-ocular, mapeamento de retina, retinografia colorida e angiofluoresceinografia.

$\mathrm{Na}$ análise das retinografias e angiofluoresceinografias avaliou-se:

- presença e coloração das estrias angióides

- presença, localização e atividade das neovascularizações de coróide (NVC)

Para análise das freqüências foi utilizado o pacote estatístico Statistical Package for Social Sciences 13.0 (SPSS ${ }^{\circledR}$ ).

\section{RESULTADOS}

Dos 317 pacientes estudados, 163 (51,5\%) eram homens. A idade média era de 57 anos com desvio-padrão de 12,43 anos, mínimo de 11 e máximo de 91 anos. Quanto à cor da pele, 209 $(66,2 \%)$ eram brancos, $91(29,0 \%)$ amarelos, $11(3,7 \%)$ negros e $3(1,1 \%)$ pardos.

As EA eram bilaterais em todos os pacientes, e de 631 olhos pesquisados, $348(55,1 \%)$ tinham EA de coloração marrom, $153(24,2 \%)$ cinzas e $124(19,6 \%)$ marrom-avermelhadas.

No momento do diagnóstico, as acuidades visuais medidas na tabela de Snellen foram piores que 20/200 em 40,4\% dos olhos.

Comprovou-se a ausência de NVC em 103 (32,5\%) pacientes. As lesões unilaterais (Figura 1) estavam presentes em $112(35,3 \%)$ e bilaterais em $99(31,2 \%)$.

Quanto à localização, 242 (74,3\%) eram maculares, 45 (13,8\%) extra-maculares e $38(11,7 \%)$ mistas.

\section{DISCUSSÃO}

O principal achado em nossa casuística de 317 casos atendidos em 30 anos é que, apesar dos inúmeros tratamentos realizados ao longo dos anos, o prognóstico visual dos pacientes é, na maioria das vezes, pobre. A justificativa é a grande incidência de NVC que ocorre em 72 a $86 \%$ dos pacien-

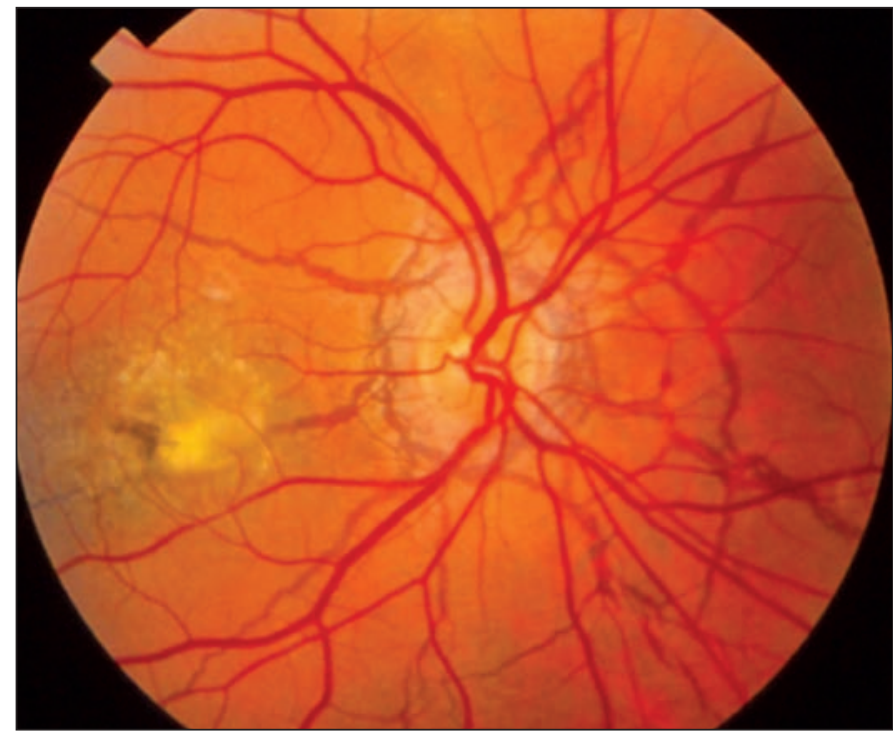

Figura 1 - Aspecto fundoscópico das estrias angióides associadas à neovascularização de coróide cicatrizada

tes implicando em baixa AV central ${ }^{(7)}$. Em nosso estudo observamos $67,6 \%$ de NVC ativas ou cicatrizadas.

O grande problema são as alterações estruturais da membrana de Bruch com degeneração de sua camada elástica associada a calcificações e microroturas que predispõem à formação de NVC sub-retiniana. O índice de recidivas é muito alto a despeito dos vários tratamentos surgidos nessas três décadas.

Em grande número dos pacientes, seu diagnóstico passa despercebido e muitas vezes é a presença de outros sinais que nos faz encontrar as estrias.

Quanto aos aspectos epidemiológicos, a distribuição das EA nos pacientes estudados não mostrou preferência quanto ao sexo. A idade média foi de 57 anos com desvio-padrão de 12,43 anos, e incidiu preferencialmente em brancos $66,2 \%$. Estes dados são semelhantes aos achados de Mansour et al. ${ }^{(8)}$ em 1993.

Quanto às características das estrias, Federman et al. ${ }^{(9)}$ encontraram bilateralidade em $100 \%$ dos casos e uma combinação de cinza e marrom na coloração de $45 \%$ das estrias. Nossos achados foram semelhantes com $92,3 \%$ bilaterais, porém com $79,58 \%$ das lesões divididas entre as cores marrom e cinza.

Durante três décadas, o tratamento com a fotocoagulação a laser foi o tratamento de escolha para as NVC, sobretudo naquelas com localização extrafoveais ${ }^{(10)}$.

Piro et al. ${ }^{(4)}$ encontraram que após a fotocoagulação $73 \%$ dos pacientes deterioraram a visão a ponto de ficarem legalmente cegos.

Clarkson et al. ${ }^{(11)}$ observaram os mesmos resultados visuais, porém em todos os pacientes estudados. As séries de casos, geralmente pequenas face à raridade da patologia, dificultam as conclusões.

A despeito disso, a fotocoagulação mostrou taxas de recorrência na ordem de $77 \%$, ocorrendo geralmente nos primeiros três meses ${ }^{(12)}$. 
Com o advento de novos horizontes no tratamento das NVC, sobretudo na degeneração macular relacionada à idade, miopia e histoplasmose ocular, muitos autores começaram a estender as novas terapias aos pacientes de EA.

Shaikh et al. ${ }^{(13)}$ usando a terapia fotodinâmica com Verteporfirina (PDT) observaram uma ação mais efetiva nas NVC subfoveais e justafoveais, porém concluíram que o PDT não altera o curso natural da doença. Destacaram ainda, altas taxas de recorrência quando comparadas às demais causas de NVC.

Costa et al. ${ }^{(14)}$ realizaram fotocoagulação seletiva do vaso nutridor obtendo resultados interessantes, porém com casuística e acompanhamentos pequenos.

As características intrínsecas do tecido nas EA é que justificam a dificuldade no tratamento das NVC desta doença.

As EA associadas a outras patologias constituem importante causa de cegueira legal devido às lesões disciformes maculares/extramaculares causadas pela $\mathrm{NVC}^{(11)}$.

Seu diagnóstico é importante, pois a despeito dos vários recursos terapêuticos das NVC, o índice de recidivas é elevado devido à patologia básica ocorrer na parte elástica da membrana de Bruch. A precocidade do diagnóstico correto e a instituição do tratamento quando necessário são fundamentais para melhora do prognóstico visual destes pacientes. Essa casuística apresentada, ímpar em seu grande número, nos ensina a procurar as estrias e tratá-las com um seguimento rigoroso.

\section{ABSTRACT}

Purpose: To evaluate the presence of choroidal neovascularization $(\mathrm{CN})$ and their sequels in patient bearing angioid streaks (AE). Methods: Case records of patients assisted from 1976 to 2006 at the Instituto Suel Abujamra located in the city of São Paulo, Brazil, were analyzed retrospectively. Age, skin color; patient complaints at the first evaluation, visual acuity, fundoscopic aspects with special attention to disciform lesions to active or scarred subretinal $\mathrm{CN}$ were considered. Results: Among the 317 studied patients, 163 (51.5\%) were men. The average age was 57 , with standard deviation of 12.4 years, minimum 11 and maximum 91 years. Regarding skin color, $209(66.2 \%)$ were white, $91(29.0 \%)$ were yellow, 11 (3.7\%) were black, and $3(1.1 \%)$ were mulatto. AE were bilateral in all patients; of 631 researched eyes, 348 (55.1\%) had brown color $\mathrm{AE}$, in $153(24.2 \%)$ they were gray and in $124(19.6 \%)$ reddish brown. Upon diagnosis, visual acuities measured with the
Snellen chart were worse than $20 / 200$ in $40.4 \%$ of the eyes. CN absence was proven in 103 (32.5\%) patients. The unilateral lesions were present in $112(35.3 \%)$ and bilateral lesions were present in 99 (31.2\%). Regarding location, 242 (74.3\%) were macular, 45 (13.8\%) were extramacular and 38 (11.7\%) were both macular and extramacular. Conclusion: AE are an important cause of legal blindness and can be easily bypassed in an ophthalmic routine examination in the absence of active or disciform neovascular lesions. The expressive number of these cases brings us to the importance of this study. It highlights the need for a meticulous fundoscopic examination in order to improve diagnoses of the disease and early treatment of $\mathrm{CN}$.

Keywords: Angioid streaks; Retinal diseases; Retina/pathology

\section{REFERÊNCIAS}

1. Green WR. Pathology of angioid streaks. In: Spencer WH, editor. Ophthalmic pathology: an atlas and textbook. $3^{\text {rd }}$ ed. Philadelphia: W.B. Saunders; 1985-1986. V.2. p.1023-32.

2. Dhermy P. Histologie de stries angioides. In: Coscas G, Soubrane G, eds. Néovasseaux Sous - Rétiniens et Laser. Paris, France: Doin Éditeurs; 1987. p.210-1.

3. Gass JDM. Angioid streaks. In: Gass JDM. Stereoscopic atlas of macular diseases: diagnosis and treatment. St Louis: Mosby; 1997. p.118-23.

4. Piro PA, Scheraga D, Fine SL. Angioid streaks: natural history and visual prognosis. In: Fine SL, Owens SL, editors. Management of retinal vascular and macular disorders. Baltimore: Willians \& Wilkins; 1983. p.136-9.

5. Doyne RW. Choroidal and retinal changes: the result of blows on the eyes. Trans Ophthalmol Soc U K. 1989;9:128.

6. Pece A, Avanza P, Galli L, Brancato R. Laser photocoagulation of choroidal neovascularization in angioid streaks. Retina. 1997;17(1):12-6.

7. Shields JA, Federman JL, Tomer TL, Annesley WH Jr. Angioid streaks. I. Ophthalmoscopic variations and diagnostic problems. Br J Ophthalmol. 1975; 59(5):257-66.

8. Mansour AM, Ansari NH, Shields JA, Annesley WH Jr, Cronin CM, Stock EL. Evolution of angioid streaks. Ophthalmologica. 1993;207(2):57-61.

9. Schields JA, Federman JL, Tomer TL, Annesley WH Jr. Angioid streaks. I. Ophthalmoscopic variations and diagnostic problems. Br J Ophthalmol. 1975; 59(5):257-66.

10. Offret G, Coscas G, Orsoni-Dupont C. Photo-coagulation des stries angioides aprés angiographie fluorescéinique. Arch Ophtalmol Rev Gen Ophtalmol. 1970; 30(5):419-22.

11. Clarkson JG, Altman RD. Angioid streaks. Surv Ophthalmol. 1982;26(5):235-46.

12. Lim JI, Bressler NM, Marsh MJ, Bressler SB. Laser treatment of choroidal neovascularization in patients with angioid streaks. Am J Ophthalmol. 1993; 116(4):414-23.

13. Shaikh S, Ruby AJ, Williams GA. Photodynamic therapy using verteporfin for choroidal neovascularization in angioid streaks. Am J Ophthalmol. 2003; 135(1):1-6. Comment in: Am J Ophthalmol. 2003;136(3):580-2; author reply 582-3.

14. Costa RA, Calucci D, Cardillo JA, Farah ME. Selective occlusion of subfoveal choroidal neovascularization in angioid streaks by using a new technique of ingrowth site treatment. Ophthalmology. 2003;110(6):1192-203. 